\title{
Was kümmert uns das Controlling in China und Indien?
}

Die Internationalisierung des Geschäfts schreitet weiter voran. Diese Entwicklung betrifft auch das Controlling.

Bei einem Blick "nach draußen" dachte man in der Vergangenheit zunächst an die weit entwickelten angelsächsischen Volkswirtschaften. Unter dem Terminus des Management Accounting haben sich in den USA und Großbritannien ausgebaute Controllingfunktionen etabliert. Dennoch findet sich der Begriff bzw. die Stelle des Controllers dort deutlich seltener als in den D-A-CH-Staaten. Dies hat nicht nur terminologische Gründe. Management Accountants verfolgen zwar durchaus ähnliche grundsätzliche Zielsetzungen, wie es etwa das neue Mission Statement der IMA (The Association for Accountants and Financial Professionals in Business, der entsprechenden amerikanischen berufsständischen Vereinigung) zeigt. Dennoch unterscheiden sie sich in mehrfacher Hinsicht von Controllern:

(1) Ihr Aufgabenspektrum ist eher enger gefasst und stärker zahlenorientiert als das "unserer" Controller.

(2) Management Accountants sind weniger stark zentralisiert organisiert; oft findet sich für sie kein eigenes Department, sondern sie sind in der Organisation "verstreut".

(3) Auf Grund des häufigen Fehlens eines wirtschaftswissenschaftlichen Studiums haben Management Accountants eine eigene berufsständische Ausbildung, die mit einem speziellen Titel (Chartered bzw. Certified Management Accountant) abschließt. Für "unsere" Controller besteht hingegen keine Verpflichtung für ein vorgegebenes Set an Fähigkeiten und Fertigkeiten. Sie weisen zumeist - nicht zwingend - eine Hochschulausbildung auf, die allerdings keiner Standardisierung unterliegt.

Aus dieser Unterschiedlichkeit und der gleichzeitigen Internationalisierungstendenz ergeben sich praktische Probleme: Soll es im Unternehmen ein einheitliches "deutsches" Controlling geben oder wird man den globalen Anforderungen eher durch ein Nebeneinander unterschiedlicher Controllingstile gerecht? Wie groß dürfen die Unterschiede sein? Wie verläuft die Abgrenzung zu bestehenden Standards? Ist das Prägen des Controllingverständnisses insbesondere in Emerging Countries zielführend?

All diese Fragen führen auf eine zentrale Problemstellung hin: Besteht noch die Möglichkeit eines spannenden Wettbewerbs der Ideen oder sind die Märkte schon verteilt? IMA und die englische Schwesterorganisation CIMA (Chartered Institute of Management Accountants) sind in diesen Märkten schon sehr sichtbar vertreten. So müssen z. B. alle koreanischen staatlichen Institutionen per Gesetz eine Balanced Scorecard Kaplan'scher Prägung einführen! Die CIMA kann dabei auf der kolonialen Tradition Großbritanniens aufbauen, wie das in diesem Heft enthaltene Beispiel Indiens zeigt. Aber auch in den Ländern Zentraleuropas, wie z. B. Polen, ist ein starker Einfluss festzustellen, beginnend bei den Textbooks für die akademische Ausbildung. Wird dieser Ideenwettbewerb kampflos aufgegeben, bestünde nun die große Gefahr, dass unser Controlling auch im "Heimatmarkt" der D-A-CH-Staaten "unter die Räder kommt". Schließlich wird es für einen nationalen Sonderweg beim Zusammenwachsen der Volkswirtschaften keinen Platz mehr geben.

Deshalb ist es unserer Überzeugung nach sehr gut investierte Zeit, zunächst einmal mehr Wissen über das Thema zu erwerben. Hierzu haben wir in diesem Heft einen bunten Reigen von Artikeln aus unterschiedlichen Ländern und einer Mischung von Theorie und Praxis zusammengestellt. Unser Ziel ist es, die Internationalisierung des Controllings überhaupt auf die Agenda der Controller zu holen. Damit wollen wir seiner wichtigen strategischen Bedeutung gerecht werden - vom sportlichen Ehrgeiz, dem bei uns sehr erfolgreichen Controlling auch international zu mehr Bedeutung zu verhelfen, einmal ganz abgesehen!

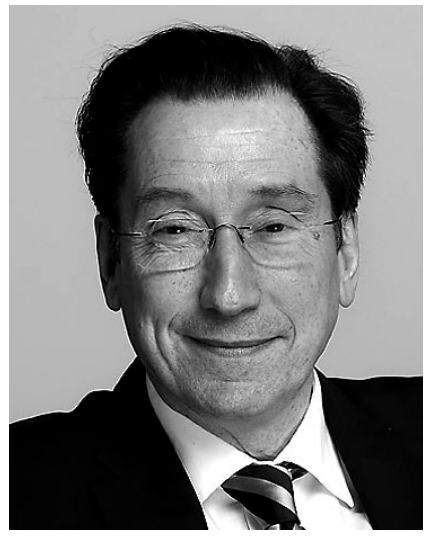

Jürgen Weber

Viel Spaß boim Lesen.

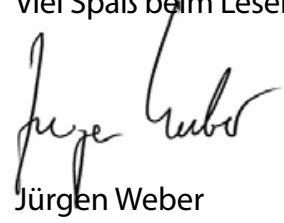

